\title{
An Easy Road to Multi-contra-classicality
}

\author{
Luis Estrada-González ${ }^{1,2}$ (D)
}

Received: 28 July 2020 / Accepted: 18 September 2021

(c) The Author(s), under exclusive licence to Springer Nature B.V. 2021

\begin{abstract}
A contra-classical logic is a logic that, over the same language as that of classical logic, validates arguments that are not classically valid. In this paper I investigate whether there is a single, non-trivial logic that exhibits many features of already known contra-classical logics. I show that Mortensen's three-valued connexive logic M3V is one such logic and, furthermore, that following the example in building M3V, that is, putting a suitable conditional on top of the $\{\sim, \wedge, \vee\}$-fragment of $\mathbf{L P}$, one can get a logic exhibiting even more contra-classical features.
\end{abstract}

\section{Introduction}

Until very recently, most of the more well-known non-classical logics-constructive logics, relevance logics, paraconsistent logics, and so on-were subclassical. Contra-classical logics are logics with valid arguments that are invalid in classical logic over the same underlying language. ${ }^{1}$ Examples of such contra-classical logics are Aristotelian syllogistic - as it validates Subalternation, Darapti or Camestrosor connexive logics, which validate Aristotle's, that is, $\sim(A \rightarrow \sim A)$ and $\sim(\sim A \rightarrow A)$, and Boethius' Theses, that is, $(A \rightarrow B) \rightarrow \sim(A \rightarrow \sim B)$ and $(A \rightarrow \sim B) \rightarrow \sim(A \rightarrow B)$.

No contra-classical logics are subclassical, even if they can be built upon some subclassical logics. Wansing (2005) obtained the connexive logic $\mathbf{C}$ by modifying the falsity condition for the conditional in Nelson's logic $\mathbf{N 4}$, with the feature that the converses of Boethius' Theses also hold. Then Omori (2016) used the same idea, changing the falsity condition for the conditional on top of LP to get another connexive logic, dLP. After that, he has shown (cf. Omori Unpublished typescript) that a number of well-known and new paraconsistent and relevant logics can be obtained

\footnotetext{
${ }^{1}$ This is "superficial contra-classicality" in the sense of Humberstone in Humberstone (2000). I consider "profound contra-classicality" - that is, non-translatability into a fragment of classical logic—as an excessive demand, but discussing that goes beyond the scope of this paper.
}

Luis Estrada-González

loisayaxsegrob@gmail.com

1 Institute for Philosophical Research, UNAM, Mexico City, Mexico

2 Department of Logic, Nicolaus Copernicus University in Toruń, Toruń, Poland 
also by changing appropriately the falsity condition for some connectives while leaving the FDE-like truth and falsity conditions for the remaining ones, in most cases even negation, fixed. ${ }^{2}$ More recently, Wansing and Unterhuber (2019) modified the falsity condition of Chellas' basic conditional logic $\mathbf{C K}$ and they obtained a (weakly) connexive logic. Even more recently, Omori and Wansing (2019) have put forward a systematization of connexive logics based on certain controlled modifications in the conditional's truth and falsity conditions, showing that, in general, changing the truth condition has led only to weak connexivity (Boethius' Theses hold only in rule form, if at all), whereas changing the falsity condition has led to hyper-connexivity (i.e. not only do Boethius' Theses hold, but also their converses).

Let me call that general approach to non-classical logic 'the Bochum Plan', in analogy with the Australian, American and Scottish plans for relevance. But the analogy can only go so far, because the scope of the Bochum Plan is far broader than paraconsistency and relevance, as the connexive logics obtained show. In fact, Omori and Wansing (2018) have generalized that idea of modifying a falsity condition to get other contra-classical logics, not only connexive logics in the ballpark of Wansing's C and Omori's dLP. Roughly, the recipe they followed for crafting a contra-classical logic from a given logic $\mathbf{L}$ is as follows:

- Provide a (model-theoretic) semantics for $\mathbf{L}$, in the FDE-like style;

- take at least one connective, leave its truth condition fixed and change its falsity condition by making it almost the falsity condition of some other connective in L;

- leave the truth and falsity conditions for the remaining connectives fixed.

What counts as "almost the falsity condition of another connective" is decidedly vague. However, I hope it is clear in the examples below, where the falsity conditions are the usual ones for other connectives. Other, more familiar non-classical logics, can be obtained by making less radical changes. ${ }^{3}$

One striking and to some extent unexpected outcome obtained by modifying the falsity condition of the conditional a la Wansing is that the logics obtained are not only connexive but also contradictory (or negation-inconsistent) because they validate formulas of the forms $X$ and $\sim X$. Thus, the modifications in the falsity condition for the conditional might induce the presence of different brands of contra-classicality into a single logic. This is important because the different sorts of contra-classicality are, in general, logically independent. For example, connexive logics in the early contemporary era-for example, those in Angell (1962), McCall

\footnotetext{
${ }^{2}$ There are plenty presentations of Belnap and Dunn's FDE and of González-Asenjo's/Priest's logic LP; the reader can choose their favorite ones but the relational ones as found since Dunn (1976) and Priest (1980), respectively, would be particularly helpful.

${ }^{3}$ In Omori (2017) there is a particularly nice example that not any modification in the evaluation conditions, not even a large number of them, produces contra-classical logics. For example, if one tweaks the falsity conditions of all of $\sim, \wedge, \vee$ and $\rightarrow$ in FDE in certain ways, one ends up with Sette's $\mathbf{P}^{1}$, which is not contra-classical, but subclassical (paraconsistent) at the atomic level and classical for molecular formulas. See Estrada-González (2020a) for a more thorough discussion of the Bochum Plan and its scope.
} 
(1966), Pizzi (1977, 1991) and Priest (1999)—were not contradictory. ${ }^{4}$ Or consider Abelian logics, logics that validate

$$
\begin{array}{ll}
(A \rightarrow A) & \text { Identity } \\
(A \rightarrow A) \leftrightarrow \sim(A \rightarrow A) & \text { Centering } \\
((A \rightarrow B) \rightarrow B) \rightarrow A & \text { Meyer-Slaney Relativity }
\end{array}
$$

and which are contradictory but not connexive. ${ }^{5}$ And even if Abelian logics also combined two sorts of contra-classicality-contradictoriness and relativity-, Meyer and Martin's logic SI $\mathbf{I}$ in Meyer and Martin (2019) also validates both of Identity and Centering without validating Meyer-Slaney Relativity.

Let $C_{1}, \ldots, C_{n}$ be the sets of defining properties of each certain $n$ kinds of contraclassicality. For the most part, I will assume that, for any $C_{j}$ and $C_{k}$ such that $j \neq k$, $C_{j} \nsubseteq C_{k}$. This is primarily because of the following two reasons. First, there remains a (limit) case of contra-classicality in the form of triviality and, should we assign an index $C_{i}$ to triviality, we would immediately have that for some $C_{j}, C_{j} \subseteq C_{i}$. Also, when studying the better-known kinds of contra-classical logics (viz. connexive, Abelian, negation-inconsistent), an underlying implicit assumption is that they must be non-trivial to be of philosophical interest. Thus, in keeping with this implicit assumption, the expectation is that there be multi-contra-classical logics that incorporate as many non-standard validities as possible while staying non-trivial. A logic $\mathbf{L}$, which for simplicity will be understood as a collection of valid arguments, is then multi-contra-classical if it satisfies three or more of the $C_{n}$ 's, none of which corresponds to triviality in this paper.

The question I am interested in here is, then, how far one could go into multicontra-classicality, beyond connexivity and contradictoriness, by changing the conditional alone. ${ }^{6}$ I start by studying a logic exhibiting such multi-contra-classicality already offered in the connexive literature: Mortensen's three-valued connexive logic, from Mortensen (1984). It is not only connexive and contradictory, its two more well-known properties, but also has important overlaps with Abelian logic and it validates $\sim(A \rightarrow B)$. Then, I will show that a logic exhibiting even more contraclassical features than Mortensen's logic can be obtained by a simple application of the Bochum Plan: take $\mathbf{L P}$ and keep all of it fixed except the falsity condition for the conditional; change it to the falsity condition of conjunction and voilà!, a logic exhibiting more than the usual number of contra-classical features arises. Because one is able to obtain Mortensen's conditional by an application of the Bochum Plan, it belongs to a family of modified conditionals put on top of the implicationless

\footnotetext{
${ }^{4}$ Furthermore, they were obtained in a way that can be described as a modification of the truth condition for the conditional, not the falsity condition.

${ }^{5}$ Basic references on Abelian logics include Casari $(1989,1997)$ and Meyer and Slaney $(1989,2002)$.

${ }^{6}$ In Francez (2020), another route to multi-contra-classicality—called 'poly-connexivity' - is presented. That route, however, is more intricate - it is achieved by modifying the falsity condition of more than one connective-and the resulting logic matches no contra-classical logic in the previous literature. It can be useful in modelling certain phenomena like intonational stress, though. See Francez (Unpublished typescript) for more information.
} 
fragment of LP. A couple of members of this family are going to be discussed in some detail too.

The plan for the rest of the paper is as follows. In Sect. 2, I present Mortensen's three-valued connexive logic, $\mathbf{M 3 V}$, at tutorial speed. In Sect. 3, I compare the M3V conditional with other two conditionals typically added on top of the $\{\sim, \wedge, \vee\}$-fragment of LP (or of a logic including such fragment). In passing, I make some original comments on what can one learn from those conditionals about hyper-connexivity and Kapsner strength, the latter a property suggested as a stronger demand logics should satisfy to better capture the core intuitions behind the notion of connexivity. Then, in Sect. 4 I present one further variation for the conditional, not yet studied in the literature, which yields even more contra-classical features than $\mathbf{M 3 V}$, and discuss its philosophical significance beyond the already interesting feature of exhibiting multi-contra-classicality.

Two final disclaimers are in order. Most of the logics, and especially the conditionals, studied here, have already well-known applications to ordinary language issues, and I mention some of them here and there. Nonetheless, my study is almost purely formal (which does not make it less philosophical!); applications of the notions introduced here are left for another occasion. Second, the paper abounds with names of connectives, principles and logics. Keeping track of them might be a bit tiring, but this is unavoidable to some extent: part of the import of the present investigation is to make explicit connections between several topics hitherto developed independently.

\section{Mortensen's Three-Valued Connexive Logic}

The logic M3V was introduced, although not with that name, in Mortensen (1984) (the name was given in McCall (2012), presumably to mean "Mortensen's 3-valued connexive logic"). The following truth tables, with $V_{\mathbf{M 3 V}}=\{2,1,0\}$ and $D^{+}=\{2,1\}$, characterize $\mathbf{M} 3 \mathbf{V}$ :

\begin{tabular}{llllll}
\hline$A$ & $B$ & $\sim A$ & $A \wedge B$ & $A \vee B$ & $A \rightarrow B$ \\
\hline 2 & 2 & 0 & 2 & 2 & 1 \\
2 & 1 & 0 & 1 & 2 & 0 \\
2 & 0 & 0 & 0 & 2 & 0 \\
1 & 2 & 1 & 1 & 2 & 1 \\
1 & 1 & 1 & 1 & 1 & 1 \\
1 & 0 & 1 & 0 & 1 & 0 \\
0 & 2 & 2 & 0 & 2 & 1 \\
0 & 1 & 2 & 0 & 1 & 1 \\
0 & 0 & 2 & 0 & 0 & 1 \\
\hline
\end{tabular}

A biconditional can be defined as usual, that is, as $(A \rightarrow B) \wedge(B \rightarrow A)$. It must be noted that Mortensen's satisfiability conditions for the conditional are structurally the same as the ones used by Anderson and Belnap in Anderson and Belnap (1975) 
to show the consistency of the logic $\mathbf{E}$ and, in particular, to show how to block the paradox of necessity, i.e. to avoid validating formulas of the form $X \rightarrow(Y \rightarrow Z)$, where $X$ is a contingent truth and $(Y \rightarrow Z)$ is a logical truth. ${ }^{7}$

The three-valued nature of Mortensen's logic, along with the number of elements in $D^{+}$and the evaluation conditions for negation motivate the representation of Mortensen's 2, 1, 0 as three subsets of the set of classical values $\{1,0\}$, namely $\{1\},\{1,0\}$ and $\{0\}$, respectively, leaving the remaining subset $\varnothing$ aside as in the twovalued relational semantics for $\mathbf{L P}$ :

\begin{tabular}{llllll}
\hline$A$ & $B$ & $\sim A$ & $A \wedge B$ & $A \vee B$ & $A \rightarrow B$ \\
\hline$\{1\}$ & $\{1\}$ & $\{0\}$ & $\{1\}$ & $\{1\}$ & $\{1,0\}$ \\
$\{1\}$ & $\{1,0\}$ & $\{0\}$ & $\{1,0\}$ & $\{1\}$ & $\{0\}$ \\
$\{1\}$ & $\{0\}$ & $\{0\}$ & $\{0\}$ & $\{1\}$ & $\{0\}$ \\
$\{1,0\}$ & $\{1\}$ & $\{1,0\}$ & $\{1,0\}$ & $\{1\}$ & $\{1,0\}$ \\
$\{1,0\}$ & $\{1,0\}$ & $\{1,0\}$ & $\{1,0\}$ & $\{1,0\}$ & $\{1,0\}$ \\
$\{1,0\}$ & $\{0\}$ & $\{1,0\}$ & $\{0\}$ & $\{1,0\}$ & $\{0\}$ \\
$\{0\}$ & $\{1\}$ & $\{1\}$ & $\{0\}$ & $\{1\}$ & $\{1,0\}$ \\
$\{0\}$ & $\{1,0\}$ & $\{1\}$ & $\{0\}$ & $\{1,0\}$ & $\{1,0\}$ \\
$\{0\}$ & $\{0\}$ & $\{1\}$ & $\{0\}$ & $\{0\}$ & $\{1,0\}$ \\
\hline
\end{tabular}

Applying the mechanical procedure described in Omori and Sano (2015) for turning truth tables employing three of the four truth values of FDE into Dunn conditions (i.e., pairs of positive and negative conditions in terms of containing or not containing the classical values 0 or 1 ), we define a relation $\sigma$, which takes formulas as its domain and the set of truth values $\{1,0\}$ as its codomain.

Then, the positive condition describes the cases in which $1 \in \sigma(X)$, and the negative condition describes the cases in which $0 \in \sigma(X)$. From the truth tables above we can infer that the conditions for the implication-free fragment of the language are standard, and that the clauses for $\rightarrow$ are as follows:

- $1 \in \sigma(A \rightarrow B)$ if a and only if $1 \notin A$, or $0 \notin B$, or both $0 \in A$ and $1 \in B$

- $0 \in \sigma(A \rightarrow B)$ if and only if $[1 \in \sigma(A)$ or $0 \in \sigma(A)]$ and $[1 \in \sigma(B)$ or $0 \in \sigma(B)]$

Finally, logical validity in $\mathbf{M 3 V}$ is understood in the usual, forwards truth-preservation way:

- $\Gamma F_{\mathrm{M3V}} A$ if and only if, for all $\sigma$, if $1 \in \sigma(B)$ for all $B \in \Gamma$ then $1 \in \sigma(A)$

We are now in a position to point out some of M3V's main features. First, we can easily see that M3V validates unrestricted Detachment, i.e.

\footnotetext{
7 A logic containing M3V was developed around the same time by Peña to cope with comparatives, gradables and vagueness. See Peña (1995) for a summary of his results and Paoli (2006) for a more friendly exposition of them.
} 


$$
A, A \rightarrow B \vDash_{\mathrm{M3V}} B
$$

unlike $\mathbf{L P}$, which validates only restricted Detachment, i.e.

$$
\text { If } k A \text { and } \vDash A \rightarrow B \text { then } \vDash B
$$

It is well-known that $\mathbf{M 3} \mathbf{V}$ is connexive, i.e.

$$
\begin{array}{ll}
\mathrm{F}_{\mathbf{M 3 V}} \sim(A \rightarrow \sim A) & \text { Aristotle's Thesis } \\
\mathrm{F}_{\mathbf{M 3 V}} \sim(\sim A \rightarrow A) & \text { Variant of Aristotle's Thesis } \\
\mathrm{F}_{\mathbf{M 3 V}}(A \rightarrow B) \rightarrow \sim(A \rightarrow \sim B) & \text { Boethius' Thesis } \\
\mathrm{F}_{\mathbf{M 3 V}}(A \rightarrow \sim B) \rightarrow \sim(A \rightarrow B) & \text { Variant of Boethius' Thesis }
\end{array}
$$

and

$$
\not_{\mathbf{M} 3 \mathbf{V}}(A \rightarrow B) \rightarrow(B \rightarrow A) \quad \text { Non-symmetry of implication }
$$

and that it is contradictory, i.e. there is pair of $\mathbf{M} 3 \mathbf{V}$-theorems such that one is the negation of the other, for example, $(A \wedge \sim A) \rightarrow A$ and $\sim((A \wedge \sim A) \rightarrow A)$.

One of the most overlooked features of $\mathbf{M 3 V}$ is that, in it, the falsity condition for the conditional is but a sophisticated way of expressing

$$
0 \in \sigma(A \rightarrow B)
$$

which implies that

$$
\mathrm{k}_{\mathrm{M} 3 \mathrm{~V}} \sim(A \rightarrow B)
$$

for any $A$ and $B$.

Now, it is easy to see, with a simple substitution in the consequent, that $\sim(A \rightarrow \sim B)$ is valid in M3V too. Because of this, I say that M3V is ultra-Abelardian. Claudio Pizzi has urged the connexive logic community not to multiply the principles with names of ancient philosophers. However, that plays a role in keeping a healthy logical memory. Peter Abelard held that conditionals express natures and that natures are characterized positively. For example, he believed that it would not be part of a human's nature to not be a stone, although being an animal would be. (For details see Martin 2004.) Thus, for him, no conditional of the form $A \rightarrow \sim B$, where $A$ is necessarily positive - that is, its main connective is not a negation-and $\sim B$ is not a subformula of $A$, is true on pain of contradiction. Omitting the constraints on $A$ and $\sim B$ would lead to ultra-Abelardianism.

Let me say that a logic $\mathbf{L}$ is hyper-connexive if for some of the Boethius' Theses, its converse holds as well. Although the converses were known and held by Boethius himself, they deserve another name precisely because of Wansing's efforts in stressing of the philosophical import of the falsity condition for the conditional, i.e. the circumstances under which a proposition of the form $\sim(A \rightarrow B)$ is true ${ }^{8}$ :

\footnotetext{
${ }^{8}$ Boethius' (or Wansing's) Theses strengthened into biconditional form is labelled 'full commutation of the conditional with negation' in Egré et al. (2021).
} 


$$
\begin{array}{ll}
\sim(A \rightarrow B) \rightarrow(A \rightarrow \sim B) & \text { Wansing's Thesis } \\
\sim(A \rightarrow \sim B) \rightarrow(A \rightarrow B) & \text { Variant of Wansing's Thesis }
\end{array}
$$

Given the validity of Detachment and $\sim(A \rightarrow B)$ (and Uniform Substitution), neither Wansing's Thesis nor its variant are valid in $\mathbf{M} 3 \mathbf{V}$, on pain of conditional triviality, i.e. the validity of any conditional. (For a countermodel to both the thesis and its variant, let $\sigma(A)=\sigma(B)=\{1\}$.)

Almost obvious given the validity of $\sim(A \rightarrow B)$, but even more overlooked, is the fact that M3V shares valid axiom schemas with many standard Abelian logics, namely the Centering axiom schemas:

$$
\begin{aligned}
& \mathrm{F}_{\mathbf{M 3 V}} \sim(A \rightarrow A) \\
& \mathrm{F}_{\mathbf{M 3 V}} \sim(A \rightarrow A) \leftrightarrow(A \rightarrow A)
\end{aligned}
$$

Nonetheless, it does not validate the Meyer-Slaney relativity axiom (schema), characteristic of purely implicative Abelian logics:

$$
\not \nvdash_{\mathrm{M} 3 \mathrm{~V}}((A \rightarrow B) \rightarrow B) \rightarrow A
$$

(For a countermodel, let $\sigma(A)=\{0\}$ and $\sigma(B)=\{1\}$.)

The validity of $\sim(A \rightarrow A)$ demands moreover a comparison with Meyer and Martin's SI $\sim \mathbf{I}^{9}{ }^{9}$ It is axiomatized as follows (names of the axioms are taken, as usual, from the combinatory logic literature):

Axioms

$$
\begin{aligned}
& \text { B. }(C \rightarrow D) \rightarrow((A \rightarrow C) \rightarrow(A \rightarrow D)) \\
& \text { B. }(A \rightarrow C) \rightarrow((C \rightarrow D) \rightarrow(A \rightarrow D)) \\
& \text { I. } A \rightarrow A \\
& \sim I . \sim(A \rightarrow A)
\end{aligned}
$$

\section{Rules}

(BX) If $\vDash_{\text {SI I I }} C \rightarrow D$ then $\vDash_{\text {SI I }}(A \rightarrow C) \rightarrow(A \rightarrow D)$

$\left(\mathrm{B}^{\prime} \mathrm{X}\right)$ If $\vDash_{\mathrm{SI} \boldsymbol{I}} A \rightarrow C$ then $\vDash_{\mathrm{SI} \boldsymbol{I}}(C \rightarrow D) \rightarrow(A \rightarrow D)$

(BXY) If $F_{\mathrm{SI} \sim \boldsymbol{I}} C \rightarrow D$ then, if $\mathrm{F}_{\mathrm{SI} \boldsymbol{I}} A \rightarrow C$ then $\mathrm{F}_{\mathrm{SI} \boldsymbol{I}} A \rightarrow D$

However, in a sense, M3V destroys the underlying idea behind $\mathbf{S I} \sim \mathbf{I}$. Meyer and Martin wanted to provide a logic for Aristotle's syllogistic, which was not reflexive. In their logic $\mathbf{S I} \sim \mathbf{I}, A \rightarrow A$ was treated as a borderline case, both a fallacy and a validity, hence the validity of both $A \rightarrow A$ and $\sim(A \rightarrow A)$. But transitivity, in its different manifestations, like $B$ and $B^{\prime}$, was not such a borderline case. Validating their negations, as $\mathbf{M 3 V}$ does, could be interpreted as formalizing the idea that the

\footnotetext{
9 They do not call it in that way, though. However, as they call 'S' the logic consisting of strict Detachment plus the axiom schemas B and B' below, I simply indicated what further axiom schemas are added to the basis $\mathbf{S}$.
} 
validity of transitivity is a borderline case too, something that Meyer and Martin would surely reject.

\section{M3V and Some Three-Valued Conditionals}

Comparing the M3V conditional with some non-contraposible conditionals will lead us to a better understanding of $\mathbf{M 3 V}$. The three-valued conditional defined by the following clauses

$$
\begin{aligned}
& 1 \in \sigma(A \rightarrow B) \text { if and only if } 1 \notin \sigma(A) \text { or } 1 \in \sigma(B) \\
& 0 \in \sigma(A \rightarrow B) \text { if and only if } 1 \notin \sigma(A) \text { or } 0 \in \sigma(B)
\end{aligned}
$$

is a conditional, first introduced in Olkhovikov (2016) and then introduced independently in Cantwell (2008) and Omori (2016). Here it is its truth table, symbolized with ' $A \rightarrow{ }_{O C O} B$ ', for more visibility:

\begin{tabular}{llll}
\hline$A \rightarrow_{\text {OCO }} B$ & $\{1\}$ & $\{1,0\}$ & $\{0\}$ \\
\hline$\{1\}$ & $\{1\}$ & $\{1,0\}$ & $\{0\}$ \\
$\{1,0\}$ & $\{1\}$ & $\{1,0\}$ & $\{0\}$ \\
$\{0\}$ & $\{1,0\}$ & $\{1,0\}$ & $\{1,0\}$ \\
\hline
\end{tabular}

Mortensen's conditional is just the contraposible conditional built upon the OCO conditional, i.e. $\left(A \rightarrow_{O C O} B\right) \wedge\left(\sim B \rightarrow_{O C O} \sim A\right)$, as it can be easily verified. ${ }^{10}$ For definiteness, let me call ' $\mathbf{C N}$ ' the logic resulting from adding the OCO conditional to the $\{\sim, \wedge, \vee\}$-fragment of $\mathbf{L P} .^{11}$

Consider now the conditional as defined in RM3: ${ }^{12}$

$1 \in \sigma(A \rightarrow B)$ if and only if $0 \in \sigma(A)$ or $[1 \in \sigma(B)$ and $0 \notin \sigma(B)$

$0 \in \sigma(A \rightarrow B)$ if and only if $1 \in \sigma(A)$ and $0 \in \sigma(B)$

As in the previous case, this conditional can also be turned into a conditional with contra-classical features by changing its falsity condition to the Wansing falsity condition. Then, the pair

$1 \in \sigma(A \rightarrow B)$ if a and only if $1 \notin A$, or $0 \notin B$, or $[0 \in A$ and $1 \in B]$

\footnotetext{
10 Note that one can define the OCO conditional from the Mortensen conditional as follows: $((A \rightarrow B) \vee B) \wedge \sim(A \rightarrow(\sim(A \rightarrow B) \wedge(B \wedge \sim B)))$. This observation can be found in the proof for Proposition 4.8 in Omori and Wansing (2020).

${ }^{11}$ Because it is equivalent to Cantwell's logic $\mathbf{C N}$, even if his motivations and way of presenting it conceptually were very different from this one.

12 The truth table for the RM3 conditional, from which these Dunn conditions can be obtained, can be found in Priest (2008, p. 125). For the more technical details see Anderson and Belnap (1975, p. 470).
} 
$0 \in \sigma(A \rightarrow B)$ if and only if $0 \notin \sigma(A)$ or $0 \in \sigma(B)$

defines the conditional introduced by in Belikov and Leginov (2019). The resulting truth table for $A \rightarrow_{B L} B$ is as follows:

\begin{tabular}{llll}
\hline$A \rightarrow_{B L} B$ & $\{1\}$ & $\{1,0\}$ & $\{0\}$ \\
\hline$\{1\}$ & $\{1\}$ & $\{0\}$ & $\{0\}$ \\
$\{1,0\}$ & $\{1\}$ & $\{1,0\}$ & $\{0\}$ \\
$\{0\}$ & $\{1,0\}$ & $\{1,0\}$ & $\{1,0\}$ \\
\hline
\end{tabular}

Again, Mortensen's conditional is just the contraposible conditional built upon the BL conditional, i.e. $\left(A \rightarrow_{B L} B\right) \wedge\left(\sim B \rightarrow_{B L} \sim A\right)$, as it can be easily verified. For definiteness, let me call 'dRM3' the logic resulting from adding the BL conditional to the implicationless fragment of RM3.

However, note that when either the OCO or the BL conditionals are, so to speak, the official conditional in the logic, the validity of ultra-Abelardianism and the Centering axiom schemes are lost, even if connexivity and contradictoriness are retained and hyper-connexivity is gained. An easy inspection of the evaluation conditions reveals that what tells M3V apart from CN and dRM3 is that, in M3V, $0 \in \sigma(A \rightarrow B)$ even when $\sigma(A)=\sigma(B)=\{1\}$, which is what warrants the validity of $\sim(A \rightarrow B)$ and provides the counterexamples to hyper-connexivity. On the other hand, what makes them similar is, first, that $\sigma(A \rightarrow B)=\{0\}$ when $1 \in \sigma(A)$ and $\sigma(B)=\{0\}$; second, that $0 \in \sigma(A \rightarrow B)$ when $\sigma(A)=\{0\}$. This latter fact -together with the LP negation, of course- is what brings connexivity in such a straightforward manner. $^{13}$

Kapsner has insisted that in order to meet the intuitive connexive requirements, it is not sufficient to validate Aristotle's and Boethius' Theses, but to guarantee that both $A \rightarrow \sim A$ and $\sim A \rightarrow A$ are unsatisfiable, and that for all interpretations, either $A \rightarrow B$ or $A \rightarrow \sim B$ is unsatisfiable. (See for example Kapsner 2012, 2019, 2020.) That a formula $X$ is unsatisfiable means that there is no interpretation under which it is true. Classically, this is equivalent to say that $X$ is false under all interpretations. These two ways of being unsatisfiable can come apart in non-classical contexts, though, and so there is at least two ways of cashing Kapsner's requirements out. ${ }^{14}$

Following Omori and Wansing (2020), let me say that a logic $\mathbf{L}$ is truth-relative Kapsner-strong if and only if, for no valuation $\sigma$,

(tKSa) $\quad 1 \in \sigma(A \rightarrow \sim A)$ and $1 \in \sigma(\sim A \rightarrow A)$, and

\footnotetext{
13 But making a conditional false when the antecedent is false is not a necessary condition to obtain connexivity, though. For example, McCall's CC1 from McCall (1966) is not like that. The price to pay for that, however, is using classical negation and making the conditional look very biconditional-ish.

14 This is not so uncommon. We can examine the notion of a theorem as an example. For a sentence to be a theorem, one can demand that it be true under every interpretation, or that it not be false under any interpretation. For a discussion on this difference in a connexive setting, see Estrada-González (2020b).
} 
(tKSb) $1 \in \sigma(A \rightarrow B)$ and $1 \in \sigma(A \rightarrow \sim B)$.

It was under this understanding of Kapsner-strength that $\mathbf{C N}, \mathbf{M 3 V}$ and other logics were evaluated in Estrada-González and Ramírez-Cámara (2016), and the result was that none of the contradictory logics was Kapsner-strong. However, let me say that a logic $\mathbf{L}$ is falsity-relative Kapsner-strong if and only if, for any valuation $\sigma$,

(fKSa) $\quad 0 \in \sigma(A \rightarrow \sim A)$ and $0 \in \sigma(\sim A \rightarrow A)$, and

(fKSb) $1 \in \sigma(A \rightarrow B)$ iff $0 \in \sigma(A \rightarrow \sim B)$, and $0 \in \sigma(A \rightarrow B)$ iff $1 \in \sigma(A \rightarrow \sim B)$.

When Kapsner-strength is understood in the latter way, M3V, CN and dRM3 are Kapsner-strong. ${ }^{15}$

\section{A Multi-contra-classical Variant of LP}

We have seen that $\mathbf{M 3 V}$ is a logic that encompasses many contra-classical features in a single framework. It only took adding the three-valued $\mathbf{E}$ conditional to the $\{\sim, \wedge, \vee\}$-fragment of $\mathbf{L P}$ to get that. We saw other non-trivial contra-classical logics - that is, logics that are both connexive and contradictory-extending the $\{\sim, \wedge, \vee\}$-fragment of $\mathbf{L P}$ with a conditional connective; in some cases, this meant changing the falsity condition of other well-known conditionals.

A natural question at this point is whether there is a non-trivial extension of the $\{\sim, \wedge, \vee\}$-fragment of $\mathbf{L P}$ which validates even more kinds of contra-classicality. For example, whether it can validate the characteristic axiom schemas of the following kinds of logics:

- Relativity logics: $((A \rightarrow B) \rightarrow B) \rightarrow A$ Meyer-Slaney Relativity

- Lovelight logics: Schemas of the form $\Lambda\left(R_{T}\right)$

where $R_{T}$ is any theorem of a relativity logic and, assuming that the language includes not only a conditional but also negation, conjunction and disjunction, $\Lambda(-)$ is defined as follows:

\footnotetext{
15 In Omori and Wansing (2020), the authors observe that M3V is Kapsner-strong if satisfiability is understood as negative satisfiability, i.e. a formula $A$ is negatively satisfiable iff there is an interpretation in which it is not false. Note that (fKSa) above requires the (negative) unsatisfiability of $A \rightarrow \sim A$ and the (negative) unsatisfiability of $\sim A \rightarrow A$.

I must confess that I feel some uneasiness about defining Kapsner-strength negatively, perhaps because in that case there would be theorems that are nonetheless (falsity-relative) unsatisfiable, producing some cheap contradictions. I feel a similar dissatisfaction when a logical truth is defined not as a formula that is true under all interpretations but as one that it is never false: what was being defined was logical truth, not logical non-falsity. I am ready to separate the notions of logical truth and theorem, but not to conflate logical truth and logical non-falsity.
} 


$$
\begin{aligned}
& \Lambda(p)=p \\
& \Lambda(\sim A)=\sim \Lambda(A) \\
& \Lambda(A \odot B)=(\Lambda(A) \subseteq \Lambda(B)), \text { for } \odot \in\{\wedge, \vee\} \\
& \Lambda(A \rightarrow B)=(\Lambda(B) \rightarrow \Lambda(A))
\end{aligned}
$$

\section{- Super-contracting logics: $(A \rightarrow(A \rightarrow B)) \rightarrow B$ Super-absorption}

Some remarks regarding these kinds of logics are in order. First, the mainstream approach to relativity logics has been adding the Relativity axiom schema to a basic purely implicative logic and then enriching the resulting logic. Nonetheless, the earliest study of a relativity logic in Meredith and Prior (1968) used, together with Substitution and Detachment, the single axiom

$$
(A \rightarrow B) \rightarrow(((C \rightarrow B) \rightarrow A) \rightarrow C \quad \text { Meredith's Axiom }
$$

Second, one can take Meyer and Slaney's idea that the Relativity axiom schema is a generalized version of the arrow version of Double Negation Elimination and then obtain relativity logics from relativizing a certain collection $\mathrm{N}$ of basic principles concerning negation. ${ }^{16}$

Third, whether the Meyer-Slaney relativization will lead to contra-classicality or not will unsurprisingly depend on the principles about negation one starts with. Suppose that one starts with $A \rightarrow A$ and $A \vee \sim A$, such that the latter is the only $\mathrm{N}_{i}$. Then one ends up with $A \rightarrow A$ and $A \vee(A \rightarrow B)$, both of which are classically valid.

Finally, if a relativity logic is required to be based on BCI logic, as it is suggested since Meyer and Slaney (2002), then Assertion is provable and hence super-contracting logics would be a special case of lovelight ones. ${ }^{17}$

16 Consider for example, as in Schechter (2005, Chapter 20), the four arrow versions of Contraposition and also the arrow versions of Double Negation Introduction and Elimination as our $\mathrm{N}$ and then relativize them. That is, take each of

$\begin{array}{lrr}\text { (N1) } & (A \rightarrow \sim B) \rightarrow(B \rightarrow \sim A) & \text { Contraposition 1 } \\ \text { (N2) } & A \rightarrow \sim \sim A & \text { Double Negation introduction } \\ \text { (N3) } & (A \rightarrow B) \rightarrow(\sim B \rightarrow \sim A) & \text { Contraposition 2 } \\ \text { (N4) } & \sim \sim A \rightarrow A & \text { Double Negation elimination } \\ \text { (N5) } & (\sim A \rightarrow B) \rightarrow(\sim B \rightarrow A) & \text { Contraposition 3 } \\ \text { (N6) } & (\sim B \rightarrow \sim A) \rightarrow(A \rightarrow B) & \text { Contraposition 4 }\end{array}$

and then apply the Meyer-Slaney relativization, defined as follows:

$\varrho\left(N_{i}\right)=N_{i}[A \rightarrow X / \sim A]$, for all strict subformulas $A$ of $N_{i}$ and a unique $X$ not a subformula of $N_{i}$.

The result of which is three purely implicative theorems classically valid plus three contra-classical theses:

$\begin{array}{lrr}\text { (RN1) } & (A \rightarrow(B \rightarrow C)) \rightarrow(B \rightarrow(A \rightarrow C)) & \text { Permutation } \\ \text { (RN2) } & A \rightarrow((A \rightarrow C) \rightarrow C) & \text { Assertion } \\ \text { (RN3) } & (A \rightarrow B) \rightarrow((B \rightarrow C) \rightarrow(A \rightarrow C)) & \text { Suffixing } \\ \text { (RN4) } & ((A \rightarrow B) \rightarrow B) \rightarrow A & \text { Meyer-Slaney Relativity } \\ \text { (RN5) } & ((A \rightarrow C) \rightarrow B) \rightarrow(B \rightarrow C) \rightarrow A & \text { Meredith's Permutation } \\ \text { (RN6) } & ((B \rightarrow C) \rightarrow(A \rightarrow C)) \rightarrow(A \rightarrow B) & \text { Suffix cancellation }\end{array}$

17 A good starting point regarding BCI logic and, in general, contemporary uses of combinatory logic, is Humberstone (2011, Ch. 2). 
Let me return to the question whether there is a way to expand the $\{\sim, \wedge, \vee\}$-fragment of LP to get more kinds of contra-classicality-for example, those just mentioned-into a single logic. The answer is affirmative. Actually, it only takes modifying the falsity condition of the $\mathbf{L P}$ conditional as follows:

$$
0 \in \sigma\left(A \rightarrow_{O} B\right) \text { iff } 0 \in \sigma(A) \text { or } 0 \in \sigma(B)
$$

In terms of the Bochum Plan, this move amounts to substituting the falsity condition of the conditional with the falsity condition of conjunction. Here it is the truth table for more visibility:

\begin{tabular}{llll}
\hline$A \rightarrow_{O} B$ & $\{1\}$ & $\{1,0\}$ & $\{0\}$ \\
\hline$\{1\}$ & $\{1\}$ & $\{1,0\}$ & $\{0\}$ \\
$\{1,0\}$ & $\{1,0\}$ & $\{1,0\}$ & $\{1,0\}$ \\
$\{0\}$ & $\{1,0\}$ & $\{1,0\}$ & $\{1,0\}$ \\
\hline
\end{tabular}

Let me call 'mccLP' the logic resulting from adding the $\mathrm{O}$ conditional to the $\{\sim, \wedge, \vee\}$-fragment of LP. ${ }^{18}$ Like its LP-like relatives in the previous sections, mccLP is contradictory — as witnesses, take $(A \wedge \sim A) \rightarrow A$ and $\sim((A \wedge \sim A) \rightarrow A)$ and although it is (falsity-relative) Kapsner strong, it is not properly hyper-connexive, because it fails the Non-symmetry of Implication. ${ }^{19}$

Validating this many contra-classical theses surely comes with a cost. Indeed, the failure of Non-symmetry of Implication might be seen as one of them. Moreover, the following features of the $\mathrm{O}$ conditional might suggest that we are no longer dealing with a connective recognizable as a conditional: ${ }^{20}$

- $\mathrm{F}_{\mathbf{m c c L P}}\left(A \rightarrow_{O} B\right) \rightarrow_{O}(A \wedge B)$

- $A, A \rightarrow_{O} B \not \nvdash_{\text {mccLP }} B$, including the strict version, when both premises and conclusions are theorems. For a counterexample, consider the schema

$$
\left.\left.\left(\left(\left(A \rightarrow_{O} \sim A\right) \rightarrow_{O} \sim A\right)\right) \rightarrow_{O}\left(A \rightarrow_{O} \sim A\right) \rightarrow_{O} \sim A\right)\right) \rightarrow_{O}\left(A \rightarrow_{O} \sim A\right)
$$

\footnotetext{
18 The name 'mccLP' stands for 'multi-contra-classical LP'. The O conditional is named after Hitoshi Omori; he suggested in conversation this particular modification of the negative clause. This connective has a twin, defined with the same truth and falsity conditions but over the $\{\sim, \wedge, \vee\}$-fragment of $K_{3}$, and rediscovered many times during the twentieth-century. See Egré et al. (2021) for both historical and logical discussion, and Estrada-González and Ramírez Cámara (2020) for further discussion of the apparently odd properties of that connective, which resembles a lot the discussion below about the conditionality of the $\mathrm{O}$ conditional.

19 The only countermodel that could do the job would be $\sigma(A)=\{0\}$ and $\sigma(B)=\{1\}$, but that gives $\sigma(A \rightarrow B)=\{1,0\}$, which makes $\sigma((A \rightarrow B) \rightarrow(B \rightarrow A))=\{1,0\}$ as well.

20 This happens because the $\mathrm{O}$ conditional satisfies the conditions to face the trilemma in Egré et al. (2021): if $\sigma(A \rightarrow B) \neq\{0\}$ when $\sigma(B)=\{0\}$ and $1 \in \sigma(A)$ or neither $1 \in \sigma(A)$ nor $0 \in \sigma(A)$, then such a conditional is symmetric, or it fails Detachment, or it fails Identity, regardless of the notion of logical consequence chosen to evaluate it.
} 
which is a logical truth in mccLP; moreover, its antecedent is but an instance of Identity, which is a logical truth in the logic too, but the consequent is not.

- Now, given Dwyer's Theorem, ${ }^{21}$ the invalidity of strict Detachment implies that some of the rules (BX), ( $\left.\mathrm{B}^{\prime} \mathrm{X}\right)$ and (BXY) must be invalid in mccLP as well, indicating that the conditional is not fully transitive. In fact, only (BXY) is invalid in mccLP. For a counterexample to (BXY), consider

$$
\left(\left(A \rightarrow_{O} \sim A\right) \rightarrow_{O} A\right) \rightarrow_{O}\left(A \rightarrow_{O} \sim A\right)
$$

and

$$
A \rightarrow_{O}\left(\left(A \rightarrow_{O} \sim A\right) \rightarrow_{O} A\right)
$$

These two are logical truths in mccLP, however, $\left.A \rightarrow_{O}\left(A \rightarrow_{O} \sim A\right)\right)$ is not. Moreover,

$$
A \rightarrow_{O} B, B \rightarrow_{O} C \not \nvdash_{\text {mecLP }} A \rightarrow_{O} C
$$

Let me briefly explain why I do not think that these are insurmountable problems. First, equivalence between two formulas $X$ and $Y$ comes in many degrees: one has

- the validity of a biconditional, $X \leftrightarrow Y$, most likely defined as $(X \rightarrow Y) \wedge(Y \rightarrow X)$

- inter-derivability, i.e. $X \dashv \vdash Y$;

- generalized equivalence, i.e. $1 \in \sigma(X)$ iff $1 \in \sigma(Y)$, and $0 \in \sigma(X)$ iff $0 \in \sigma(Y)$, for all $\sigma$;

- strict equivalence, that is, $\sigma(X)=\sigma(Y)$, for all $\sigma$.

Thus, even if one has $\vDash_{\text {mccLP }}(A \rightarrow B) \leftrightarrow(A \wedge B)$, ones does not have any of interderivability (since $A \rightarrow B \not \nvdash_{\mathbf{m c c L P}} A \wedge B$ ), generalized or strict equivalence: simply consider the case when $\sigma(A)=\{1,0\}$ and $\sigma(B)=\{0\}$. Hence, contrary to the appearances, and in spite of having many conjunctive-like features, the $\mathrm{O}$ conditional does not collapse into a conjunction.

One can be alarmed because of the validity of a certain form of Simplification: $\left(A \rightarrow_{O} B\right) \rightarrow_{O} A$ and $\left(A \rightarrow_{O} B\right) \rightarrow_{O} B$. Nonetheless, one should give reasons for thinking of the arrow as a conjunction in the antecedent but as a conditional when it is the main connective. If one does not apply that double standard here and thinks that $A \rightarrow_{O} B$ is uniformly a conjunction, it validates something not very conjunction-like (let me use ' $\otimes$ ' as a generic conjunction): $(A \otimes B) \otimes A$ and $(A \otimes B) \otimes B$. (Several forms of transitivity, to be discussed below, do not make sense for a conjunction, either.)

So much for the danger of ending with a more conjunction-like connective. Let me give now some reasons to think that we are still in the presence of a conditional. First, the failure of Detachment, even in the strict form, might appear as a serious

21 A logic $\mathbf{L}$ validating the axiom schemas $\mathrm{B}$ and $\mathrm{B}^{\prime}$ validates the rules $(\mathrm{BX}),\left(\mathrm{B}^{\prime} \mathrm{X}\right)$ and $(\mathrm{BXY})$ if and only if it validates (strict) Detachment. See Martin (1978, Appendix A) for a proof. 
drawback for some people. Nonetheless, Beall has stressed several times (see for example Beall 2011, 2015) that even if Detachment is invalid for the conditional in $\mathbf{L P}$, it is default valid in the sense that $A, A \rightarrow B \vDash_{\mathbf{L P}} B \vee(A \wedge \sim A)$ holds, that is, either Detachment holds or the antecedent is a formula evaluated as $\{1,0\}$, which arguably is not the case in most situations. The second disjunct internalizes in the conclusion the structure of truth values into the object language: Detachment does not hold when the antecedent is both true and false, linguistically expressed as $A \wedge \sim A$. Thus, either Detachment holds for $A$ and $A \rightarrow B$, or $A$ is both true and false, i.e. $A \wedge \sim A$. Detachment is default valid exactly in the same way in mccLP, that is, $A, A \rightarrow_{O} B \vDash_{\text {mccLP }} B \vee(A \wedge \sim A)$.

Second, in the very formulation of the final worry already comes the answer. Even if transitivity of the $\mathrm{O}$ conditional is lost in some forms, it is kept in others, for example in the validity of $(\mathrm{BX})$ and $\left(\mathrm{B}^{\prime} \mathrm{X}\right)$. Additionally, the arrow forms of $(\mathrm{BX})$, $\left(\mathrm{B}^{\prime} \mathrm{X}\right)$ and $(\mathrm{BXY})$ hold. But perhaps more importantly, Beall's strategy to recover Detachment as default valid in LP can be borrowed to recover (BXY) and plain Transitivity, since their invalidity is due to the contradictoriness of the middle term. Thus,

$$
A \rightarrow_{O} B, B \rightarrow_{O} C \mathrm{~F}_{\mathbf{m c c L P}}\left(A \rightarrow_{O} C\right) \vee(B \wedge \sim B)
$$

If $k_{\text {mccLP }} C \rightarrow D$ then, if $k_{\text {mccLP }} A \rightarrow C$ then $k_{\text {mccLP }}(A \rightarrow D) \vee(C \wedge \sim C)$

Third: as it has already been advanced since Footnote 16, mccLP is the same logic as the one named DF/TT in Egré et al. (2021), in the sense that they validate exactly the same set of valid arguments. The authors did not notice all its contraclassical potential, though-besides connexivity, they just discussed the validity of $\left(A \rightarrow_{O} B\right) \rightarrow_{O} A$. Moreover, it is a bit mistaken identifying the $\mathrm{O}$ conditional with the "De Finetti" conditional: the latter is undetermined, has neither truth value, when the antecedent is not true, whereas the former is overdetermined, has both truth values, when the antecedent is not simply true. To cope with logical consequence in a sensible manner, the "De Finetti" conditional needs to be paired with preservation of non-falsity, whereas the $\mathrm{O}$ conditional can be paired with the usual preservation of truth. Although those differences do not alter the set of arguments validated, it does affect the presentation and proper understanding of all the features of the logic, not reducible to the arguments validated.

This leads me to discuss the issue of the philosophical import of mccLP more generally and beyond the philosophy of logic. As I have already said, that so many contra-classical features can coexist in a single logic is already worth mentioning. Nonetheless, this might not be seen as a good feature, though, for the different contra-classical features might differ in degree of plausibility. For example, (hyper-) connexivity - and, surprisingly, contradictoriness- seems better motivated than, say, lovelight features, or the simplification of conditionals. ${ }^{22}$

I agree with the above remark. Nonetheless, when facing weird yet new or understudied logics, my working assumption is that, if the logic is non-trivial and it

$\overline{22}$ I want to thank an anonymous referee for urging me to discuss this point. 
contains a bunch of other recognizable principles about the usual connectives, it is very unlikely to get certain principles-contra-classical theses in this case-validated just by chance. Then my working methodology in those cases is that a creative, more charitable stare towards the formalism is needed; not everything should be left to the founding parents nor everything should be judged empty or meaningless without having tried really hard to get something from the apparent dryness. Of course, sometimes this would be an exercise of trying to get blood from a stoneand not everyone should be devoted to do that-, but sometimes the stone might in fact be a lithops. I still do not know whether mccLP is a creature more attractive than a lithops, but I am almost sure that it is not a stone.

The above is not flashy rhetoric. DF/TT in Egré et al. (2021) has been extensively studied as a logic suitable for suppositional reasoning, that is, as a logic where conditionals are read as expressing conditional degrees of belief. Thus, mccLP at least opens the door for studying suppositional reasoning from the point of view of inconsistent degrees of belief. But the possible applications are not exhausted there. $A \rightarrow_{O} B$ belongs to two ancient and venerable traditions:

- that of non-vacuously true conditionals, conditionals that are not true (only) when their antecedent is not true, ${ }^{23}$

- that of comparative conditionals, where ' $A \rightarrow B$ ' is read ' $B$ is comparable to $A$ (according to some parameter), ${ }^{24}$

That the conditional is related to these two issues opens further avenues of investigation. Moreover, highlighting the multi-contra-classicality of mccLP points towards a common feature of conditional negation, categorial grammar and constructive falsification-which deliver hyper-connexivity - comparative conditionals - the main application of relativity logics - , suppositional reasoning - which delivers simplification of the conditional-and subjunctive conditionals - one of the contemporary homes of connexivity; see Angell $(1962,2016)$ - This is not to say that all these phenomena can be handled at once with mccLP, but merely that they might have important common features that deserves further exploration.

To conclude this section, let me mention one more conditional in this family. From certain remarks in Peña (1995) one can consider an extreme case of a comparative conditional that can be read " $B$ is at least as true as $A$, provided they have different values and $A$ is not false":

\begin{tabular}{llll}
\hline$A \rightarrow_{P} B$ & $\{1\}$ & $\{1,0\}$ & $\{0\}$ \\
\hline$\{1\}$ & $\{1,0\}$ & $\{1,0\}$ & $\{0\}$ \\
$\{1,0\}$ & $\{1,0\}$ & $\{1,0\}$ & $\{1,0\}$
\end{tabular}

\footnotetext{
23 See for example Egré and Cozic (2016, Section 7) and Douven (2016, Chapter 2) for general discussions of the topic and further references. Note that the Mortensen, OCO and Belikov and Leginov's conditionals are restricted cases of this; they are not true (only) when the antecedent is false (only).

24 See for example Casari (1989) and Peña (1995), the useful comparison of those two views in Paoli (2006) and the already mentioned application in Paoli (1999).
} 


\begin{tabular}{llll}
\hline$A \rightarrow_{P} B$ & $\{1\}$ & $\{1,0\}$ & $\{0\}$ \\
\hline$\{0\}$ & $\{0\}$ & $\{1,0\}$ & $\{1,0\}$ \\
\hline
\end{tabular}

Put on top of the $\{\sim, \wedge, \vee\}$-fragment of $\mathbf{L P}, A \rightarrow_{P} B$ delivers almost the same as $A \rightarrow_{O} B$, with a big difference: it is ultra-Abelardian, i.e. $\left(A \rightarrow_{P} \sim B\right)$ becomes valid, as in M3V.

Note that this invites comparative readings of the previous conditionals as follows:

$A \rightarrow{ }_{O C O} B:$ " $B$ is at least as true as $A$, provided $A$ is not false";

$A \rightarrow_{B L} B:$ : $B$ is at least as true as $A$, provided both $B$ and $A$ are not false";

Note that the provisos may play the same role as suppositions in suppositional conditionals, and that the different conditionals express different reactions to the unsatisfaction of the provisos or the suppositions. Again, this resembles the situation of conditionals with just true antecedents. Nonetheless, a more precise and systematic study of both the non-vacuously true conditionals in the context of contra-classical logics, and of the contra-classical logics following Peña's ideas on comparative conditionals, is left for further work.

\section{Conclusion}

I started with the question whether there is a way to expand the $\{\sim, \wedge, \vee\}$-fragment of LP to get more kinds of contra-classicality, not only connexivity and contradictoriness, into a single logic. The answer is affirmative. Mortensen's three valued connexive logic $\mathbf{M 3 V}$, which can be described as putting the three-valued $\mathbf{E}$ conditional on top of the aforementioned fragment, was an early example of that.

After discussing many properties of $\mathbf{M 3 V}$, especially in comparison with other connexive and contra-classical logics, I introduced a logic exhibiting even more contra-classical features than $\mathbf{M 3 V}$. It can be obtained by a simple application of the Bochum Plan: take LP, keep all of it fixed except the falsity condition for the conditional and change it to the falsity condition of conjunction, giving raise to a logic dubbed 'mccLP'.

The present study leaves open at least the following research avenues:

- The treatment of multi-contra-classicality in a more general setting, for example, on top of FDE rather than LP;

- an integrated study of the different topics that usually motivate certain contraclassical logics and whose formal counterparts coexist in mccLP, namely, suppositional reasoning, conditional negation or comparative conditionals;

- a more systematic study of non-vacuously true conditionals in the context of contra-classical logics. 
Acknowledgements This paper was written during the COVID-19 crisis. I want to thank the PAPIIT project IN403719 and the Nicolaus Copernicus University in Toruń (Excellence Initiative - Research University Program, Application 437) for the financial support. I also want to thank Antonio Recio for the many laughs during this difficult period, and Hitoshi Omori and Elisángela Ramírez-Cámara for several useful discussions surrounding the $\mathrm{O}$ conditional. Elisángela and Alejandro Estrada-Girón helped me to make the typescript more readable. I benefited from useful comments from the audience at the $L P-40$ Years After conference held in Bochum in November 2019, where the first ancestor of this paper was presented. I want to thank the organizers, Heinrich Wansing and Hitoshi Omori, for the invitation and generous support. I also got valuable comments from Federico Pailós, Damián Szmuc, Elia Zardini and other attendees to the Online Seminar on Iberoamerican Logic. Finally, I want to thank the anonymous referees for their corrections and suggestions to improve the typescript.

\section{References}

Anderson, A. R., \& Belnap, N. D. (1975). Entailment: The logic of relevance and necessity (Vol. I). Princeton: Princeton University Press.

Angell, R. B. (1962). A propositional logic with subjunctive conditionals. Journal of Symbolic Logic, 27(3), 327-343.

Angell, R. B. (2016). Connexive implication, modal logic and subjunctive conditionals. IFCoLog Journal of Logics and their Applications, 3(3), 297-308.

Beall, J. (2011). Multiple-conclusion LP and default classicality. Review of Symbolic Logic, 4(2), 326-336.

Beall, J. (2015). Free of detachment: Logic, rationality, and gluts. Noûs, 49(2), 410-423.

Belikov, A., \& Leginov, E. (2019). Dummett's truth theory and connexive logic (Unpublished typescript).

Cantwell, J. (2008). The logic of conditional negation. Notre Dame Journal of Formal Logic, 49(3), $245-260$.

Casari, E. (1989). Comparative logics and Abelian $\ell$-groups. In R. Ferro, C. Bonotto, S. Valentini, \& A. Zanardo (Eds.), Handbook of the history of logic (pp. 101-246). Elsevier.

Casari, E. (1997). Conjoining and disjoining on different levels. In M. L. D. Chiara, K. Doets, D. Mundici, \& J. van Benthem (Eds.), Logic and scientific methods. Volume one of the Tenth Congress of Logic, Methodology and Philosophy of Science, 1995 (pp. 261-287). Kluwer.

Douven, I. (2016). The epistemology of indicative conditionals: Formal and empirical approaches. Cambridge University Press.

Dunn, J. M. (1976). Intuitive semantics for first-degree entailments and 'coupled trees'. Philosophical Studies, 29(3), 149-168.

Egré, P., \& Cozic, M. (2016). Conditionals. In M. Aloni \& P. Dekker (Eds.), The Cambridge handbook of formal semantics (pp. 490-524). Cambridge University Press.

Egré, P., Rossi, L., \& Sprenger, J. (2021). De Finettian logics of indicative conditionals Part I: Trivalent semantics and validity. Journal of Philosophical Logic, 50, 187-213.

Estrada-González, L. (2020a). The Bochum Plan and the foundations of contra-classical logics. CLE e-Prints, 19(1), 1-22.

Estrada-González, L. (2020b). Possibility, consistency, connexivity. In S. Negri, G. Sandu, N. Olivetti, \& R. Verbrugge (Eds.), Advances in modal logic (Vol. 13, pp. 189-207). College Publications.

Estrada-González, L., \& Ramírez-Cámara, E. (2016). A comparison of connexive logics. IFCoLog Journal of Logics and their Applications, 3(3), 341-355.

Estrada-González, L., \& Ramírez-Cámara, E. (2020). Non-conditional contracting connectives. In S. Rahman, M. Mojtahedi \& M. S. Zarepour (Eds.) Mathematics, logics and their philosophies. Essays in Honor of Mohammad Ardeshir (pp. 349-364).

Francez, N. Poly-connexivity: Connexive conjunction and disjunction (Unpublished typescript).

Francez, N. (2020). A poly-connexive logic. Logic and Logical Philosophy, 29(1), 143-157.

Humberstone, L. (2000). Contra-classical logics. Australasian Journal of Philosophy, 78(4), 438-474.

Humberstone, L. (2011). The connectives. MIT Press.

Kapsner, A. (2012). Strong connexivity. Thought: A Journal of Philosophy, 1(2), 141-145.

Kapsner, A. (2019). Humble connexivity. Logical and logical. Philosophy, 28(3), 513-536. 
Kapsner, A. (2020). Connexivity and the pragmatics of conditionals. Erkenntnis. https://doi.org/10.1007/ s10670-020-00325-1.

Martin, C. J. (2004). Logic. In J. E. Brower \& K. Guilfoy (Eds.), The Cambridge Companion to Abelard (pp. 158-199). Cambridge University Press.

Martin, E. (1978). The P-W problem. PhD thesis, Australian National University.

McCall, S. (1966). Connexive implication. Journal of Symbolic Logic, 31(3), 415-433.

McCall, S. (2012). A history of connexivity. In D. M. Gabbay, F. J. Pelletier, \& J. Woods (Eds.), Handbook of the history of logic. Vol. 11. Logic: A history of its central concepts (pp. 415-449). Elsevier.

Meredith, C. A., \& Prior, A. N. (1968). Equational logic. Notre Dame Journal of Formal Logic, IX(3), 212-226.

Meyer, R., \& Martin, E. (2019). S revisited. Australasian Journal of Logic, 16(3), 49.

Meyer, R. K., \& Slaney, J. K. (1989). Abelian logic (from A to Z). In R. Routley, G. Priest, \& J. Norman (Eds.), Paraconsistent logic: Essays on the inconsistent (pp. 245-288). Philosophia.

Meyer, R. K., \& Slaney, J. K. (2002). A, still adorable. In W. Carnielli, Í. D’Ottaviano, \& M. Coniglio (Eds.), Paraconsistency: The logical way to the inconsistent (pp. 245-288). Marcel Dekker.

Mortensen, C. (1984). Aristotle's thesis in consistent and inconsistent logics. Studia Logica, 43(1-2), $107-116$.

Olkhovikov, G. K. (2016). On a new three-valued paraconsistent logic. In Logic of law and tolerance (pp. 96-113). Ural State University Press, Yekaterinburg, 2001 (in Russian). English version in IfColog Journal of Logics and their Applications, 3(3), 317-334.

Omori, H. Towards a systematic overview of paraconsistent logics (Unpublished typescript).

Omori, H. (2016). From paraconsistent logic to dialetheic logic. In H. Andreas \& P. Verdée (Eds.), Logical studies of paraconsistent reasoning in science and mathematics (pp. 111-134). Springer.

Omori, H. (2017). Sette's P ${ }^{1}$ logics, revisited. In A. Baltag, J. Seligman, \& T. Yamada (Eds.), Logic, rationality, and interaction. 6th International Workshop LORI 2017, Sapporo, Japan, September 11-14, 2017, proceedings (pp. 451-465). Springer.

Omori, H., \& Sano, K. (2015). Generalizing functional completeness in Belnap-Dunn logic. Studia Logica, 103, 883-917.

Omori, H., \& Wansing, H. (2018). On contra-classical variants of Nelson logic N4 and its classical extension. Review of Symbolic Logic, 11(4), 805-820.

Omori, H., \& Wansing, H. (2019). Connexive logic. An overview and current trends. Logic and Logical Philosophy, 28(3), 371-387.

Omori, H., \& Wansing, H. (2020). An extension of connexive logic C. In S. Negri, G. Sandu, N. Olivetti, \& R. Verbrugge (Eds.), Advances in modal logic (Vol. 13, pp. 503-522). College Publications.

Paoli, F. (1999). Comparative logic as an approach to comparison in natural language. Journal of Semantics, 16(1), 67-96.

Paoli, F. (2006). Comparing two views of comparison: Peña and Casari on vagueness. Logic and Philosophy of Science, IV(1), 105-121.

Peña, L. (1995). Lógicas multivaluadas. In C. A. Alchourrón, J. M. Méndez, \& R. Orayen (Eds.), Enciclopedia Iberoamericana de Filosofía. Vol. 7: Lógica (pp. 323-349). Trotta.

Pizzi, C. (1977). Boethius' thesis and conditional logic. Journal of Philosophical Logic, 6(1), 283-302.

Pizzi, C. (1991). Decision procedures for logics of consequential implication. Notre Dame Journal of Formal Logic, 32(4), 618-636.

Priest, G. (1980). Sense, entailment and Modus Ponens. Journal of Philosophical Logic, 9(4), 415-435.

Priest, G. (1999). Negation as cancellation, and connexive logic. Topoi, 18(2), 141-148.

Priest, G. (2008). An introduction to non-classical logics. From if to is (2nd ed.). Cambridge University Press.

Schechter, E. (2005). Classical and nonclassical logics: An introduction to the mathematics of propositions. Princeton University Press.

Wansing, H. (2005). Connexive modal logic. In R. Schmidt, I. Pratt-Hartmann, M. Reynolds, \& H. Wansing (Eds.), Advances in modal logic (Vol. 5, pp. 367-383). College Publications.

Wansing, H., \& Unterhuber, M. (2019). Connexive conditional logic. Part I. Logic and Logical Philosophy, 28(3), 567-610.

Publisher's Note Springer Nature remains neutral with regard to jurisdictional claims in published maps and institutional affiliations. 